\title{
Effect of acyl chain composition on salt-induced lamellar to inverted hexagonal phase transitions in cardiolipin
}

\author{
M.B. Sankaram ${ }^{1}$, Gary L. Powell ${ }^{2}$ and Derek Marsh ${ }^{1}$ \\ ' Max-Planck-Institut für biophysikalische Chemie, Abteilung Spekttroskopie. Göningen (F.R.G.) \\ and ${ }^{2}$ Department of Biological Sciences. Clemson University. Clemson. SC (U.S.A.)
}

(Recelved 17 Nonember 1988)

Key words: Cardiolipin; Diphosphatidylglycerol: NMR. ${ }^{31}$ P-: Non-lamellar phase; Hexagonal $\mathbf{H}_{11}$ phase

Salt-induced fluid lamellar $\left(L_{\alpha}\right)$ to inverted hexagonal $\left(H_{n}\right)$ phase transitions have been studied in diphosphatidylglycerols (cardiolipins) with different acyl chain compositions, using ${ }^{31} \mathbf{P}$ nuclear magnetic resonance (NMR) spectroscopy. Cardiolipins with four myristoyl chains, tetramyristoyl cardiolipin (TMCL), and with four oleoyl chains, tetraoleoyl cardiolipin (TOCL), were synthesized chemically. TMCL was found to undergo a thermotropic lamellar gel to lamellar liquid-crystalline phase transition at $33-35^{\circ} \mathrm{C}$. This lipid exhibited an axially symmetric ${ }^{31} \mathrm{P}-\mathrm{NMR}$ spectrum corresponding to a lamellar phase at all $\mathrm{NaCl}$ concentrations between 0 and $6 \mathrm{M}$. In the case of TOCL, formation of an $\mathrm{H}_{\mathrm{n}}$ phase was induced by salt concentrations of $3.5 \mathrm{M} \mathrm{NaCl}$ or greater. These observations, taken together with earlier findings that bovine heart cardiolipin aqueous dispersions adopt an $\mathrm{H}_{\mathrm{n}}$ phase at salt concentrations of $1.5 \mathrm{M} \mathrm{NaCl}$ or greater, indicate that increasing unsaturation and length of the acyl chains favour formation of the $\mathrm{H}_{\mathrm{n}}$ phase in diphosphatidylglycerols.

Diphosphatidylglycerols, commonly known as cardiolipins (CL), are a class of phospholipids found at high concentration in the inner mitochondrial membrane [1-3]. Extracted CL from bovine heart, when dispersed in aqueous media, exhibits a transition from a lamellar $\left(L_{\alpha}\right)$ to an inverted hexagonal $\left(H_{11}\right)$ phase which is induced by monovalent and divalent cations and by $\mathrm{pH}$ titration [4-6]. The $\mathrm{L}_{\alpha}$ to $\mathrm{H}_{\mathrm{u}}$ phase transformation is also observed in other phospholipids and is induced by various environmental conditions such as pH, temperature and salt concentration. Generally, lipid molecules with large acyl chain to headgroup volumes

Abbteviations: DMPA, 1,2-dimyristoyl-sn-glycero-3-phosphoric acid; DOPA, 1,2-dioleoyl-sn-glycero-3-phosphoric acid; DMPC, 1,2-dimyristoyl-sn-glycero-3-phosphocholine; DOPC, 1,2-dioleoyl-snglycero-3-phosphocholine; DMPG, 1,2-dimyristoyl-sn-glycero-3-phosphoglycerol; DOPG, 1,2-dioleoyl-sn-glycero-3-phosphoglycerol; CL, cardiolipin (diphosphatidylglycerol); TMCL, 1,3-bis[1,2-dimyristoylsn-glycero-3-phospho]-rac-glycerol; TOCL, 1,3-bis[1,2-dioleoyl-snglycero-3-phospho]-rac-glycerol; $\mathrm{L}_{\mathbf{a}}$ fluid lamellar phase; $\mathbf{H}_{\mathbf{I I}}$, inverted hexagonal phase; NMR, nuclear magnetic resonance: DSC, differential scanning calorimetry.

Correspondence: D. Marsh, Max-Planck-Institut für biophysikalische Chemie, Abteilung Spektroskopie, Postfach 2841, D-3400 Göttingen, F.R.G. tend to accomodate themselves better in an inverted phase than in a lamellar phase, presumably due to the packing constraints imposed on them by the latter assembly. In the specific case of cardiolipins, varying the number of acyl chains leads to a rich range of polymorphism [5]. For instance, dilysocardiolipin containing two acyl chains per two headgroup phosphates forms normal micellar aggregates in salt-free aqueous media whereas monolysocardiolipin molecules are assembled into bilayer lamellae [5]. Addition of salt induces transitions from a micellar to a lamellar phase and from a lamellar to an $\mathrm{H}_{\mathrm{n}}$ phase for dilysocardiolipin and for cardiolipin, respectively [5].

Whereas the effects of changing the number of chains in triggering phase transformations in cardiolipins have been studied in detail, the role of acyl chain composition in the induction of non-lamellar phases in cardiolipins is not clear. The influence of chainlength and chain unsaturation on the stability of non-bilayer phases has been investigated in phosphatidylethanolamines (see, for example Refs. 16 and 17). However, such studies have mostly involved thermotropically induced transitions or compositional variations. Far less is known regarding the effects on isothermal non-lamellar phase transitions triggered by varying ionic composition, especially for cardiolipins. We report here our results on calorimetric and NMR studies of salt-induced lipid

m05-2736/89/\$03.50 $\bigcirc 1989$ Elsevier Science Publishers B.V. (Biomedical Division) 
polymorphism in synthetic cardiolipins of homogeneous chain composition.

Materials and Methods. DMPG and DMPA were synthesised from DMPC (Fluka, Buchs, Switzerland), and DOPG and DOPA from DOPC (Sigma, St. Louis, $\mathrm{MO}$ ), using the headgroup exchange reaction catalysed by phospholipase D (Boehringer-Mannheim, Mannheim, F.R.G.) [7]. TMCL and TOCL were synthesised by condensing PG and PA of the appropriate chain composition, employing the method described by Keana et al. [8]. The reaction products were purified either on a Cellulose CM-52 column eluting with $\mathrm{CH}_{2} \mathrm{Cl}_{2}{ }^{\prime}$ $\mathrm{CH}_{3} \mathrm{OH} / 6 \mathrm{M} \mathrm{NH}_{4} \mathrm{OH}(65: 30: 3, \mathrm{v} / \mathrm{v})$, or by precipitation from $\mathrm{CH}_{3} \mathrm{OH}$ with hexane, or precipitation from $\mathrm{CH}_{2} \mathrm{Cl}_{2}$ with acetone. The synthetic products were characterized by thin-layer chromatography using the solvent system $\mathrm{CH}_{2} \mathrm{Cl}_{2} / \mathrm{CH}_{3} \mathrm{OH} / 6 \mathrm{M} \mathrm{NH}_{4} \mathrm{OH}(65: 30: 3$, v/v), and by high resolution ${ }^{1} \mathrm{H}-\mathrm{NMR}$ spectroscopy (see also Ref. 8).

Proton-dipolar decoupled ${ }^{31} \mathrm{P}$-NMR spectra were collected at $109 \mathrm{MHz}$ on a Bruker WH-270 spectrometer, and at $81 \mathrm{MHz}$ on an IBM spectrometer. A pulse width of $6 \mu \mathrm{s}$ and a nominal decoupling power of $20 \mathrm{~W}$ were used. To avoid sample-heating, a gated decoupling sequence was used. Typically, free induction decays were collected in 512 bytes, zero-filled to 8 Kbytes and Fourier transformed after applying a $50 \mathrm{~Hz}$ exponential line broadening. Chemical shifts were referred to external $85 \%$ phosphoric acid. Chemical shift anisotropies $\left(\Delta \sigma=\sigma_{\|}-\sigma_{\perp}\right)$ were measured from the points of maximum slope in the $\sigma_{\| 1}$ and $\sigma_{\perp}$ regions of the powder patterns corresponding to the lamellar and inverted hexagonal phases, respectively.

Differential scanning calorimetric (DSC) measurements were performed on a Perkin-Elmer DSC-2B calorimeter equipped with an Intracooler I. Samples were sealed in large-volume stainless steel pans which were heated in the calorimeter at a rate of $1.25 \mathrm{C}^{\circ} / \mathrm{min}$. The DSC pans were opened after the experiments and the samples were analysed for phosphorus content [9]. Areas under the thermograms were determined by paper weighing, and indium and benzene were used as standards to determine the transition enthalpies.

Samples for both NMR and DSC experiments were prepared by dispersing a thin film of the lipid in excess quartz-distilled water. The $\mathbf{p H}$ of the dispersions was adjusted to 7.0 with $\mathrm{NaOH}$ and $\mathrm{NaCl}$ added to the required concentration, and the samples were then centrifuged. The centrifuged pellets were then transferred to the ?'MR tubes. To eliminate small particles the NMR samples were subjected to several freeze-thaw cycles before measurement. DSC measurements were performed on a part of the NMR samples, after NMR measurement. Thin-layer chromatography was used to check that no lipid degradation had occurred during the NMK and DSC runs.
Results and Discussion. A differential scanning calorimetric trace of hydrated TMCL in $6 \mathrm{M} \mathrm{NaCl}(\mathrm{pH} 7.0)$ is given in Fig. 1. Upon heating, this lipid exhibits a thermotropic transition from a lamellar gel to a lamellar liquid-crystalline phase at a temperature of $33^{\circ} \mathrm{C}$. The enthalpy of this transition was determined to be 10.5 $\mathrm{kcal} / \mathrm{mol}(44 \mathrm{~kJ} / \mathrm{mol})$. The cooling scan shows a reversible transition at $35^{\circ} \mathrm{C}$. These data differ from those reported by Rainier et al. [10] who found that the di-ammonium salt of TMCL has a transition temperature of $40^{\circ} \mathrm{C}$ and an enthalpy of $12.5 \mathrm{kcal} / \mathrm{mol}$. A variable dependence of the transition temperature on the particular salt form of the cardiolipin was reported in this latter paper, which may explain this difference. Nagamachi et al. [18] have found an even higher transition temperature $\left(47^{\circ} \mathrm{C}\right)$ for the acid form of TMCL in distilled water.

The proton dipolar-decoupled ${ }^{31} \mathrm{P}-\mathrm{NMR}$ spectra of TMCL displayed an axially symmetric powder pattern with a chemical shift anisotropy $(\Delta \sigma)$ of $-49 \mathrm{ppm}$ at $25^{\circ} \mathrm{C}$. The spectra were essentially unchanged upon addition of salt. The lamellar phase spectrum was observed at all salt concentrations between 0 and $6 \mathrm{M}$ $\mathrm{NaCl}$.

The proton dipolar-decoupled ${ }^{31} \mathrm{P}-\mathrm{NMR}$ spectra of TOCL at $25^{\circ} \mathrm{C}$ are shown for different $\mathrm{NaCl}$ concentrations in Fig. 2. All spectra contain a small isotropic component which could be reduced, but not entirely eliminated, by repeated freeze-thawing. It is therefore likely that this component arises from small particles. The isotropic component is most evident in spectra where there is a coexistence of lamellar and $\mathrm{H}_{11}$ phases, which also suggests that it may arise from surface curvature effects. It cannot be totally excluded that this

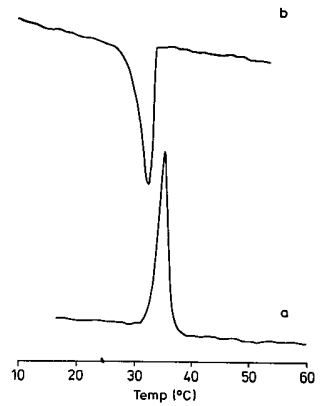

Fig. 1. Difierential scanning calorigrams of tetramyristoyl cardiolipin in $6 \mathrm{M} \mathrm{NaCl}$ ( $\mathrm{pH} \mathrm{7.0)}$ ). (a) heating scan. (b) cooling scan. Scan rate: $1.25 \mathrm{C} / \mathrm{min}$. 


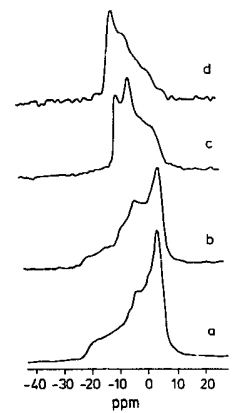

Fig. 2. $109 \mathrm{MHz}$ proton-dipolar decoupled ${ }^{31} \mathrm{P}-\mathrm{NMR}$ spectra of tetraoleoyl cardiolipin as a function of salt concentration at $25^{\circ} \mathrm{C}$. (a) $0.0 \mathrm{M} \mathrm{NaCl}$, (b) $3.0 \mathrm{M} \mathrm{NaCl}$, (c) $3.5 \mathrm{M} \mathrm{NaCl}$, and (d) $4.0 \mathrm{M} \mathrm{NaCl}$.

component may arise from a phase of cubic symmetry, but if this is the case, the amount of this phase is relatively small and will not be further considered.

When dispersed in water containing no salt, TOCL exhibits an axially symmetric powder pattern with a chemical shift anisotropy of $\mathbf{- 2 8 . 8} \mathrm{ppm}$ (Fig. 2a). This lamellar phase pattern is preserved until a salt concentration of $3.5 \mathrm{M} \mathrm{NaCl}$. At this concentration, a two-component spectrum is observed corresponding to the co-existence of a fluid lamellar phase spectrum with a $\Delta \sigma$ of $-25 \mathrm{ppm}$ and a $\mathrm{H}_{11}$ phase spectrum with a reduced chemical shift anisotropy of opposite sign: $\Delta \sigma$ $=+17$ ppm (Fig. 2c). The $H_{11}$ phase alone is then observed with increasing salt concentration until the dispersions are saturated with $\mathrm{NaCl}$. The chemical shift anisotropies of the $L_{\alpha}$ and $H_{11}$ phases for TOCL are similar to those observed previously for bovine heart cardiolipin [5], where the identity of the phases was established independently by X-ray diffraction [4].

\section{TABLE I}

${ }^{3 /}$ P.NMR chemical shift anisotropies $(\Delta \sigma)$ of cardiolipins of different chain composition, as a funrtion of salt concentration $(\mathrm{NaCl})$

\begin{tabular}{llllll}
\hline Lipid & $T$ & \multicolumn{3}{l}{$\Delta 0(\mathrm{ppm})$} & \\
\cline { 3 - 6 } & $\left({ }^{\circ} \mathrm{C}\right)$ & $0 \mathrm{M}$ & $1.5 \mathrm{M}$ & $3.5 \mathrm{M}$ & $4 \mathrm{M}$ \\
& & $\mathrm{NaCl}$ & $\mathrm{NaCl}$ & $\mathrm{NaCl}$ & $\mathrm{NaCl}$ \\
\hline TMCL & 40 & -49.9 & -49.5 & -48.7 & -48.1 \\
TOCL & 25 & -28.8 & -28.9 & $-25 .+17$ & +17.1 \\
$\begin{array}{c}\text { Bovine } \\
\text { CL }\end{array}$ & 20 & -31.8 & $-32,+19$ & +19.3 & +19.7 \\
\hline
\end{tabular}

Data from Powell and Marsh [5]. Bovine $\mathrm{Cl}$ is composed of approx. $90 \%$ linoleuyl chains [11].
The salt-induced spectral changes in the TOCL ${ }^{31} \mathbf{P}$ NMR powder patterns parallel those observed for bovine heart $\mathrm{CL}$ [5]. In the latter case, the transition to a $\mathrm{H}_{\mathrm{n}}$ phase was observed at a salt concentration of 1.5 $\mathrm{M} \mathrm{NaCl}$. The ${ }^{31} \mathrm{P}-\mathrm{NMR}$ data on bovine heart $\mathrm{CL}$, TOCL and TMCL are summarised in Table I. The previous data on bovine heart cardiolipin provide a useful comparison with the present data on synthetic cardiolipins of homogeneous chain composition, since the naturally occurring $\mathrm{CL}$ has a chain composition which approximates to that of tetralinoleoyl cardiolipin [11]. It is clear from Table I that the phase behaviour of tetraacyl $\mathrm{CL}$ is determined not simply by the unique covalent structure of this phospholipid, but also by the detailed chain composition. The short, saturated myristoyl chains favour the formation of the lamellar bilayer phase: TMCL is found here exclusively in the bilayer phase. The longer, monounsaturated oleoyl chains promote formation of the inverted hexagonal phase, in TOCL at salt concentrations of $3.5 \mathrm{M} \mathrm{NaCl}$ or greater. Increasing the degree of unsaturation from oleoyl to linoleoyl chains, further enhances the propensity for $\mathrm{H}_{\text {II }}$ phase formation by lowering the threshold salt concentration, which for bovine heart CL lies at $1.5 \mathrm{M}$ $\mathrm{NaCl}$.

Although the formation of $\mathrm{H}_{11}$ phases by the endogenous cardiolipins in biological membranes is rather unlikely under physiological conditions, the molecular properties which favour formation of these phases may well be of structural and functional significance. Cardiolipins with the potential to form $\mathrm{H}_{\mathrm{n}}$ phases may thus be important structural elements in regions of high curvature in the mitochondrial cristae, or in the contact sites with the outer mitochondrial membrane (see also Ref. 12). Incipient non-larnellar phase-forming cardiolipins may additionally possess dynamic properties which can enhance the activity of integral membrane enzymes such as cytochrome oxidase [13], as found also for unsaturated phosphatidylethanolamines in the case of the $\mathrm{Ca}^{2+}$-ATPase [14]. In connection with the present work on synthetic cardiolipins, it is also interesting to note that, in contrast to the bovine heart lipid, cardiolipin from yeast contains a high proportion of oleoyl chains, and $85 \%$ of the chains in $\mathrm{CL}$ from Acholeplasma laidlawii are composed of myristic and palmitic acids [15].

M.B.S. wishes to thank the Alexander von Humboldt Stiftung for a fellowship. G.L.P. gratefully acknowledges the support from the National Science Foundation USA-FRG Cooperative Science Program and from the National Heart, Lung \& Blood Institute (HL 38190). We gratefully acknowledge the technical assistance of D.A. Abramovitch in the synthesis of TOCL. We thank Margaret Kotun of the Department of Chemistry, Clemson University for recording some of the ${ }^{31}$ P-NMR spectra. 


\section{References}

1 loannou. P. and Golding, B.T. (1979) Prog. Lipid. Res. 17, 279-312.

2 Semin. B.K.. Saraste. M. and Wikstrom. M. (1984) Biochim. Biopitys. Acta 15, 769-782.

3 Thompson. D. and Ferguson-Miller, S. (1983) Biochemistry 22, $3178-3181$.

4 Seddon, J.M., Kaye, R.D. and Marsh, D. (1983) Biochim. Biophys. Acta 734, 347-352.

5 Powell. G.L. and Marsh, D. (1985) Biochemistry 24, 2902-2908.

6 Rand, R.P. and Sengupta, S. (1972) Biochim. Biophys. Acta 255 , 484-492.

7 Comfurius. P. and Zwaal, R.F.A. (1977) Biochim. Biophys. Acta 488, 36-42.

8 Kearna, J.F.W., Shimizu, M. and Jernstedt, K.K. (1986) J. Ög. Chem. 51, 2297-2299.

9 Eibl, H. and Lands, W.E.M. (1969) Anal, Biochem. 30, 51-57.
10 Rainier, S.. Jain, M.K., Ramirez, F., Joannou, P.V., Marecek, J.F. and Wagner, R. (1979) Biochim. Biophys. Acta 558, 187-198.

11 Powell, G.L., Knowles, P.F. and Marsh, D. (1985) Biochim. Biophys. Acta 816, 191-194.

12 Verkleij, A.J. (1984) Biochim. Biophys, Acta 779. 43-63.

13 Marsh, D. and Powell, G.L. (1988) Bioelectrochem. Bioenerg., in press.

14 Navarro, J., Toivio-Kinnucan, M. and Racker, E. (1984) Biochemistry 23, 130-135.

15 Smith. P.F., Koostra, W.L. and Henrikson, C.V. (1965) J. Bact. 90 , 282-283.

16 Seddon, J.M., Cevc, G. and Marsh, D. (1983) Biochemistry 22. 1280-1289.

17 Brown, P.M., Steers, J., Hui, S.W., Yeagle, P.L. and Silvius, J.R. (1986) Biochemistry 25, 4259-4267.

18 Nagamachi. E., Kariyama, R. and Kanemasa, Y. (1985) Physiol. Chem. Phys. Med. NMR 17, 255-260. 